Originalien

Z Gerontol Geriat 2022 · 55:305-311 https://doi.org/10.1007/s00391-021-01897-5 Eingegangen: 3. Dezember 2020

Angenommen: 31. März 2021

Online publiziert: 28. April 2021

(c) Der/die Autor(en) 2021

\section{Zusatzmaterial online}

Zusätzliche Informationen sind in der Online-Version dieses Artikels (https://doi. org/10.1007/s00391-021-01897-5) enthalten.

\section{Unser Alltag wird zunehmend digital, ob beruflich oder privat - die aktuelle Coronapandemie hat dies noch einmal verdeutlicht. Doch wie sieht die digitale Transformation in den Haushalten der Personen ab 65 Jahren aus, und wie hat sich dort die Nutzung seit 2009 verändert? Diese Kernfragen waren leitend für die hier näher vorzustellende Vergleichsanalyse auf Grundlage von 3 Bevölkerungsbefragungen aus den Jahren 2009, 2014 und 2019.}

\section{Hintergrund und Fragestellung}

Gerade in der aktuellen COVID-19Pandemie zeigte sich die Digitalisierung unseres Alltags noch einmal deutlich, im Sinne einer zunehmenden digitalen Kommunikation (z.B. durch Homeoffice). Diese zunehmende digitale Transformation zeigt sich u. a. auch im Nutzungsanstieg moderner Informations- und Kommunikationstechnologien (IKT) wie dem Internet oder dem Smartphone. Dennoch ist aus der gerontologischen Forschung bekannt, dass auch heute noch Formen der digitalen Spaltung zwischen jüngeren (unter 65 Jahre) und älteren Altersgruppen (über 65 Jahre) bestehen $[1,3]$. So zeigen Studien aus Europa, dass z. B. nur $53 \%$ der Personen ab 50 Jahren das Internet nutzen [5].

Jüngere Menschen leben heute ganz selbstverständlich in einer digitalisierten Lebenswelt. Anders ist dies bei älte-

\author{
Alexander Seifert ${ }^{1,2}$ \\ 'Zentrum für Gerontologie, Universität Zürich, Zürich, Schweiz \\ ${ }^{2}$ Fachhochschule Nordwestschweiz, Hochschule für Soziale Arbeit, Olten, Schweiz
}

\title{
Digitale Transformation in den Haushalten älterer Menschen
}

ren Personen, die mit diesen Technologien nicht groß geworden sind und somit weniger Berührungspunkte damit haben [12]. Auch wenn moderne Technologien wie z.B. eine Erinnerungshilfe auf dem Smartphone eine Hilfe im Alter darstellen können [7], zeigen Studien auch, dass älteren Menschen $z$. T. die nötigen Technikkompetenzen fehlen oder dass sie keinen direkten Vorteil in der Nutzung sehen, da sie bisher von den "klassischen Wege" wie z. B. direkten Kontakten oder dem Gang zum Bahnschalter im Alltag Gebrauch machen [16-18]. Im Alter kann sich zudem die körperliche und kognitive Funktionsfähigkeit verändern. Zudem können soziale oder finanzielle Ressourcen eingeschränkt sein oder Ängste gegenüber der Nutzung von Technik bestehen, was den Zugang zu dieser wiederum erschwert [2]. Die aktuelle COVID-19-Pandemie hat den digitalen Graben zwischen Jung und Alt noch einmal deutlicher gemacht, da Personen ohne technische Kompetenzen und Mittel fehlende direkte soziale Kontakte nicht durch technische Lösungen kompensieren können [16].

Unklar bleibt jedoch, wie weit die digitale Transformation dennoch auch in der Bevölkerungsgruppe der ab 65-Jährigen vorangeschritten ist, und inwieweit sich diese digitale Transformation der Alltagsanwendungen auf deren Einstellung zu dieser Technik generell verändert hat, bzw. ob sich auch die begründeten Faktoren einer Internetnutzung über die Jahre verändert haben. Internationale Studien [1-3, 5] liefern erste Hinweise darauf, dass einerseits beeinflussende Faktoren z.B. der Internetnutzung sich über die Jahre angepasst haben. Antworten auf diese Fragen sollen die Nutzungsprofile von Alltagstechnologien wie dem Inter- net, Smartphone oder Tablet geben. Hierbei soll anhand von deskriptiven Vergleichen bestehender Untersuchungen aus der Schweiz einerseits der zeitliche Wandel in der Techniknutzung, Technikinteresse, Anwendungsvielfalt und möglichen Hürden der Techniknutzung gegenübergestellt werden.

Diese Fragen waren Ausgangspunkt vorliegender Vergleichsstudie. Grundlage des Vergleichs liefern 3 Schweizer Querschnittserhebungen aus den Jahren 2009, 2014 und 2019; welche hier erstmals deskriptiv verglichen werden sollen, um die oben genannten zeitlichen Veränderungen darzustellen. Es soll hierbei eruiert werden: (a) wie sich die Einstellungen zur Technik und zum Internet in der Zeit von 2009 bis 2019 verändert haben, (b) wie sich die Internetnutzung und die Nutzung von Alltagstechnologien (alltägliche technische Geräte im Haushalt z. B. Radio, Mobiltelefon, Tablet) über die Jahre verändert haben, (c) welche Gründe der Nichtnutzung des Internets benannt werden, (d) welche Anwendungen im Internet über die Jahre bevorzugt werden, und (e) welche Einflussfaktoren für die Internetnutzung sich in den jeweiligen Untersuchungen ergeben.

\section{Studiendesign und Untersuchungsmethoden}

\section{Daten}

Grundlage für die Auswertung waren 3 repräsentative - im Querschnitt in der Schweiz erhobene - Bevölkerungsbefragungen von in Privathaushalten lebenden Personen ab 65 Jahren aus den Jahren 2009 [10], 2014 [17] und 


\section{Originalien}

\begin{tabular}{|c|c|c|c|c|}
\hline \multirow[b]{2}{*}{ Merkmal } & \multirow[b]{2}{*}{ Kategorien } & \multicolumn{3}{|l|}{ Prozente } \\
\hline & & $\begin{array}{l}2009 \\
(n=1105)\end{array}$ & $\begin{array}{l}2014 \\
(n=1037)\end{array}$ & $\begin{array}{l}2019 \\
(n=1130)\end{array}$ \\
\hline \multirow[t]{2}{*}{ Geschlecht } & Frauen & 54,7 & 52,7 & 51,0 \\
\hline & Männer & 45,3 & 47,3 & 49,0 \\
\hline \multirow[t]{2}{*}{ Altersgruppen } & 65-79 Jahre & 77,2 & 76,7 & 78,7 \\
\hline & $80+$ & 22,8 & 23,3 & 21,3 \\
\hline \multirow[t]{2}{*}{ Wohnsituation } & Allein lebend & 35,4 & 36,1 & 30,9 \\
\hline & Nicht allein lebend & 64,6 & 63,9 & 69,1 \\
\hline \multirow[t]{3}{*}{ Bildungsstand } & Bis Sekundarstufe I & 21,8 & 18,2 & 13,0 \\
\hline & Sekundarstufe II & 57,8 & 57,3 & 53,4 \\
\hline & Tertiärstufe & 20,3 & 24,5 & 33,6 \\
\hline \multirow{3}{*}{$\begin{array}{l}\text { Haushaltsein- } \\
\text { kommen }\end{array}$} & Bis CHF 4.000 & 40,0 & 38,1 & 29,7 \\
\hline & CHF 4001 bis 8000 & 45,1 & 44,1 & 48,7 \\
\hline & Über CHF 8.000 & 14,9 & 17,8 & 21,6 \\
\hline \multirow{5}{*}{$\begin{array}{l}\text { Subjektive Ge- } \\
\text { sundheit }\end{array}$} & Sehr gut & 25,9 & 46,9 & 44,4 \\
\hline & Eher gut & 45,8 & 30,8 & 25,5 \\
\hline & Teils, teils & 21,8 & 14,4 & 19,5 \\
\hline & Eher schlecht & 5,4 & 3,9 & 5,0 \\
\hline & Sehr schlecht & 1,1 & 3,9 & 5,6 \\
\hline
\end{tabular}

2019 [14]. Die Stichprobe im Jahr 2009 beinhaltet $n=1105$ Personen im Alter zwischen 65 und 97 Jahren $(M=73,8$; $S D=7,11)$, die Stichprobe aus dem Jahr 2014 beinhaltet $n=1037$ Personen im Alter zwischen 65 und 100 Jahren $(M=74,5$; $S D=6,96)$ und die Stichprobe aus dem Jahr 2019 beinhaltet $n=1130$ Personen im Alter zwischen 65 und 101 Jahren $(M=74,1 ; S D=6,69)$. Die Charakteristik der 3 Stichproben kann - Tab. 1 entnommen werden. Alle 3 Befragungen fanden jeweils in den Monaten August bis Oktober und in allen Sprachregionen der Schweiz statt. Die Interviews mit standardisierten Fragen wurden telefonisch („computer assisted telephone interview“, CATI) geführt und durch eine schriftliche Erhebung von Haushalten ohne Telefonanschluss ergänzt. Die Stichproben wurden mittels einer Wahrscheinlichkeitsauswahl aus der Grundgesamtheit der ständigen Wohnbevölkerung der Schweiz ab 65 Jahren bestimmt.

\section{Messinstrumente}

Es wurden folgende Variablen zum deskriptiven Vergleich genutzt (• Tab. 2):

\section{Ergebnisse}

\section{Statistische Analysen}

Neben deskriptiven Gegenüberstellungen der Verteilungen pro Erhebungsjahr wurden Vergleiche zwischen jüngere Altersgruppen (65 bis 79 Jahre) und älteren Altersgruppen (ab 80 Jahre) berücksichtigt (•Tab. 2). Zusätzlich wurde eine binär logistische Regressionsanalyse pro Jahr gerechnet. Fehlende Werte wurden listenweise eingeschlossen.

\section{Technikinteresse und Einstellung zum Internet}

Wie anhand von • Tab. 2 zu sehen ist, ergibt sich 2009 bei der ersten Aussage zur Techniknutzung „Der technische Fortschritt muss immer weitergehen" ein Mittelwert von 3,85 aufder Skala von 1 ,lehne völlig ab“ bis 5 „stimme völlig zu“. Im Jahr 2014 (M: 3,82) und 2019 (M: 3,71) hat sich dieser Mittelwert nicht maßgeblich verändert. Auch die beiden weiteren Aussagen („Ohne technische Geräte könnte ich mir mein Leben nicht mehr vorstellen“ und „Ich interessiere mich sehr für neue technische Dinge") wurden über alle Jahre hinweg ähnlich hoch bewertet, wenn auch die Aussage zum Interesse an neuen technischen Dingen etwas zurückhaltender im Vergleich zu den beiden ersten Aussagen bewertet wurde.

Neben den allgemeinen Einstellungen $\mathrm{zu}$ technischen Dingen konnte auch die Einstellung speziell zum Internet als eine spezifische Anwendung abgefragt werden. Es zeigt sich hier, dass auch die 3 Einstellungsfragen zum Internet (• Tab. 2) über die Jahre hinweg hoch bewertet wurden, so ergibt sich z.B. bei der ersten dazugehörigen Aussage („Das Internet erleichtert den Kontakt zu anderen Menschen") bei der Erhebung 2009 ein Mittelwert von 3,18, 2014 ein Mittelwert von 3,21 und 2019 ein Mittelwert von 3,28, auf der Skala von 1 ,trifft gar nicht zu“ bis 5 „trifft völlig zu“. Innerhalb der 3 Stichproben ist erkennbar, dass sich die Einstellungsfragen zum Internet hinsichtlich der Altersgruppen (65 bis 79 Jahre und 80 Jahre und älter) statistisch signifikant unterscheiden: Ältere Personen sind dem 
Internet gegenüber etwas negativer eingestellt als jüngere Personen.

\section{Nutzung von Alltagstechnologien}

96,7\% der 2009 befragten Personen gaben an, einen Fernseher zu besitzen; davon erklärten $89,0 \%$, dass sie diesen täglich nutzen (• Tab. 2). 2014 und 2019 stieg diese Zahl nicht weiter an. Eine ähnlich stabile, wenn auch leicht rückgängige Entwicklung ist bei der Nutzung von Radio und Festnetztelefon zu beobachten. Anders sieht dies bei den klassischen Mobiltelefonen aus, die mehr und mehr durch Smartphones ersetzt werden. Gerade die Verwendung von Smartphone und Tablet ist seit 2014 stark angestiegen und belief sich 2019 auf 63,7\% bzw. 40,0\% (- Tab. 2). Dennoch ist bei diesen neueren Mobilgeräten auch 2019 noch eine Differenz zwischen jüngeren und älteren Personen erkennbar: So besitzen zwar 75,2\% der 65- bis 79-Jährigen ein Smartphone und $47,3 \%$ ein Tablet, aber nur $34,6 \%$ bzw. $21,9 \%$ der mindestens 80-Jährigen.

\section{Nutzung des Internets}

Innerhalb der Erhebung 2009 nutzten $37,8 \%$ das Internet, 2019 waren es bereits $74,2 \%$. Dennoch zeigte sich auch 2019 weiterhin ein Unterschied zwischen den jüngeren ( 65 bis 79 Jahre) und älteren Altersgruppen (80+): So nutzten 2019 zwar $85,8 \%$ der ,jüngeren Älteren“ das Internet, aber nur $45,0 \%$ der mindestens 80 Jährigen. Es zeigt sich auch, dass nicht alle Onliner (Personen, die das Internet nutzen) täglich im Netz unterwegs sind; 2019 waren es 58,5\% (• Tab. 2).

Die Nutzungsvielfalt der Onliner zeigt sich insbesondere in der aktuellen Erhebung. So werden zwar v. a. weiterhin die klassischen Anwendungen wie z.B. die Informationssuche, das Schreiben von E-Mails und das Abrufen von Fahrplänen intensiv genutzt, aber immer mehr auch andere Anwendungen wie das Internetbanking oder das Onlinelesen von Zeitungen. Dennoch griff auch Ende 2019 nur weniger als die Hälfte der Personen auf das Internet zurück, wenn sie z. B. etwas kaufen wollten. Dasselbe galt für den Besuch sozialer Netzwerke (• Tab. 2).

Z Gerontol Geriat 2022 ·55:305-311 https://doi.org/10.1007/s00391-021-01897-5

(c) Der/die Autor(en) 2021

A. Seifert

\section{Digitale Transformation in den Haushalten älterer Menschen}

Zusammenfassung

Hintergrund. Nicht nur jüngere, sondern zunehmend auch ältere Menschen leben heute in einer Welt, in der digitale Alltagstechnologien ihren Alltag maßgeblich begleiten. Aber wie hat sich diese Techniknutzung in den letzten 10 Jahren verändert, und inwieweit hat sich die Einstellung gegenüber dieser Technik verändert? Um diese Fragen zu beantworten, wurden 3 querschnittliche Bevölkerungsbefragungen miteinander verglichen.

Material und Methoden. Das Datenmaterial stammt aus 3 Schweizer Befragungen (2009: $n=1105,2014: n=1037,2019: n=1130$ ) von Personen ab 65 Jahren. Diese Befragungen erfolgten jeweils als standardisiertes telefonisches Interview und wurden mit einer optionalen schriftlichen Befragung kombiniert.

Ergebnisse. Sowohl die Nutzung des Internets als auch die von mobilen Endgeräten (Smartphone, Tablet) ist von 2009 zu 2019 angestiegen. So nutzten $200937,8 \%$ der Befragten das Internet, 2019 waren es bereits $74,2 \%$. Dennoch ist weiterhin zu erkennen, dass v. a. Personen ab 80 Jahren diese Technologien seltener nutzen. Auch wenn 2019 bereits mehr unterschiedliche Internetanwendungen genutzt wurden als noch 2009 , so wurden jedoch ähnliche Gründe für die Nichtnutzung des Internets angegeben. Zu den Hauptgründen zählen Sicherheitsbedenken und Angaben, nach denen die Nutzung des Internets zu kompliziert bzw. das Erlernen des Umgangs mit dem Internet überhaupt zu hoch sei. Auch die Einstellungen zur Technik und die Faktoren, die für eine Internetnutzung sprechen, haben sich nur wenig verändert. Schlussfolgerung. Auch wenn die digitale Transformation voranschreitet, gibt es bei der Techniknutzung weiterhin Ungleichheiten. Auch wenn sich diese mehr und mehr nivellieren, so werden neue Technologien in den kommenden Jahren neue Ungleichheiten schaffen.

Schlüsselwörter

Smartphone - Tablet · Technik · IKT . Techniknutzung $\cdot$ Schweiz

\section{Digital transformation in the households of older people}

\section{Abstract}

Background. Digital technologies play an important role in people's everyday lives and this applies not just to younger people but increasingly to older people. The study examined how the use of and attitudes towards such technology have changed over the last 10 years through a comparison of 3 cross-sectional population surveys.

Material and methods. The data came from three Swiss surveys (2009: $n=1105 ; 2014$ : $n=1037 ; 2019: n=1130$ ) of people aged 65 years and over, each of which was conducted as a standardized telephone interview and combined with an optional written survey. Results. The use of both the internet and mobile devices (smartphones, tablets) increased from 2009 to 2019: in 2009, 37.8\% of the respondents used the internet, rising to $74.2 \%$ in 2019; however, people over 80 years old used these technologies less frequently. Although more and different internet resources were being used in 2019 than in 2009 , the reasons given for not using the internet were similar and included security concerns, claims that using the internet is too complicated and the belief that learning to use it is too difficult. There has also been little change in the attitudes toward technology and the factors explaining internet use. Conclusion. Even though the digital transformation is progressing, inequalities in the use of technology remain. Even when these are levelling out, new technologies will likely create new inequalities.

Keywords

Smartphone - Tablet · Technology · ICT · Technology use $\cdot$ Switzerland 
Tab. 2 Aspekte der digitalen Transformation

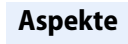

2009

2014

2019

Mittelwert (SD)

\section{Technikinteresse}

(1.1) Der technische Fortschritt muss immer weitergehen

(1.2) Ohne technische Geräte könnte ich mir mein Leben nicht mehr vorstellen

(1.3) Ich interessiere mich sehr für neue technische Dinge

Einstellung zum Internet ${ }^{2}$

(2.1) Das Internet erleichtert den Kontakt zu anderen Menschen

(2.2) Das Internet erspart viel Lauferei
(2.3) Das Internet ist anregend und faszinierend
IKT-Besitz/-Nutzung

IKT-Besitz/-Nutzung

Fernseher
Radio
Festnetztelefon
Klassisches Mobiltelefon
Smartphone
Tablet
Stationärer Computer
Laptop
Anteil der Onliner
Gründe der Nichtnutzung des Internets

Es ist zu kompliziert

Sicherheitsbedenken

Zu hoher Aufwand beim Erlernen

Eine andere Person ruft Informationen ab

Fehlende Unterstützung

Kosten

Internetanwendungen

Infosuche, allgemein

E-Mails

Fahrpläne abrufen

Gesundheitsthemensuche

Zeitungen lesen

Internetbanking

Kauf von Waren

Soziale Netzwerke

\begin{tabular}{|l|l|l|}
\hline $3,85(1,275)$ & $3,82(1,249)$ & $3,71(1,204)$ \\
\hline $3,75(1,390)$ & $3,61(1,427)$ & $3,59(1,356)$ \\
\hline $2,97(1,418)$ & $2,96(1,395)$ & $3,07(1,270)$ \\
\hline $3,18(1,551)$ & $3,21(1,550)$ & $3,28(1,487)$ \\
\hline $3,32(1,488)$ & $3,47(1,512)$ & $3,35(1,433)$ \\
\hline $3,21(1,533)$ & $3,41(1,459)$ & $3,23(1,409)$ \\
\hline 2009 & 2014 & 2019 \\
\hline
\end{tabular}

Prozente Besitz (davon tägliche Nutzung)

\begin{tabular}{l|l|l}
$96,7(89,0)$ & $95,8(92,6)$ & $95,3(87,4)$ \\
$94,4(79,9)$ & $94,7(78,9)$ & $89,6(75,9)$ \\
\hline $96,9(32,3)$ & $71,2(61,6)$ & $84,5(51,5)$ \\
\hline $76,7(16,6)$ & $71,2(30,8)$ & $47,0(43,0)$ \\
\hline- & $29,6(62,7)$ & $63,7(78,1)$ \\
\hline- & $23,9(51,1)$ & $40,0(60,3)$ \\
\hline $38,8(-)$ & $41,1(-)$ & $68,8(59,9)$ \\
\hline $23,6(-)$ & $37,3(-)$ & \\
\hline $37,8(47,2)$ & $55,7(49,8)$ & $74,2(58,5)$ \\
\hline 2009 & 2014 & 2019
\end{tabular}

Prozente (nur Offliner)

\begin{tabular}{|l|l|l|}
\hline 71,5 & 69,8 & 79,9 \\
\hline 57,2 & 62,6 & 71,1 \\
\hline 61,7 & 61,8 & 70,4 \\
\hline 46,7 & 53,7 & 66,4 \\
\hline 31,9 & 35,7 & 39,1 \\
\hline 33,9 & 37,0 & 31,9 \\
\hline 2009 & 2014 & 2019 \\
\hline
\end{tabular}

Prozente (nur Onliner)

\begin{tabular}{|l|l|l|}
\hline 81,7 & 86,5 & 93,4 \\
\hline 88,5 & 89,9 & 93,8 \\
\hline 76,1 & 75,8 & 75,8 \\
\hline 55,1 & 65,7 & 61,3 \\
\hline 46,3 & 56,5 & 57,5 \\
\hline 38,1 & 40,3 & 54,4 \\
\hline 31,0 & 43,3 & 40,7 \\
\hline 5,4 & 16,5 & 28,8 \\
\hline
\end{tabular}

\section{9}

2014

Mittelwerte nach Altersgruppen

(65-79/80+)
2019

$\begin{array}{lll}3,86 / 3,83 & 3,86 / 3,71 & 3,67 / 3,78\end{array}$

$3,79 / 3,66 \quad 3,65 / 3,49 \quad 3,65 / 3,43^{*}$

$3.09 / 2,66^{* * *} \quad 3,07 / 2,66^{* * *} \quad 3,18 / 2,80^{* * *}$

$3,24 / 3,02 \quad 3,32 / 2,91^{* * *} \quad 3,33 / 3,15$

$3,46 / 2,87^{* * *} \quad 3,57 / 3,17^{* *} \quad 3,44 / 3,10^{* *}$

$3,34 / 2,80^{* * *} \quad 3,50 / 3,13^{* *} \quad 3,37 / 2,87^{* * *}$

$20092014 \quad 2019$

Prozente nach Altersgruppen (65-79/80+)

$95,5 / 91.8 \quad 96,3 / 90,5^{* * *} \quad 92,0 / 83,4^{* * *}$

$96.6 / 97,7 \quad 95,2 / 96,5 \quad 81,1 / 94,3^{* * *}$

$87,5 / 48,8^{* * *} \quad 74,8 / 62,3^{* * *} \quad 41,0 / 61,0^{* * *}$

- $\quad 37,3 / 10,1^{* * *} \quad 75,2 / 34,6^{* * *}$

- $\quad 29,0 / 11,0^{* * *} \quad 47,3 / 21,9^{* * *}$

$47,6 / 15,9^{* * *} \quad 47,5 / 24,7^{* * *} \quad 78,4 / 44,2^{* * *}$

$29,0 / 9,6^{* * *} \quad 45,5 / 16,7^{* * *}$

$47,9 / 12,8^{* * *} \quad 67,6 / 25,4^{* * *} \quad 85,8 / 45,0^{* * *}$

$2009 \quad 2014 \quad 2019$

Prozente nach Altersgruppen (65-79/80+)

\begin{tabular}{lll}
\hline $70,4 / 73,2$ & $70,7 / 68,2$ & $77,3 / 82,2$ \\
\hline $65,9 / 42,8^{* * *}$ & $67,0 / 56,6$ & $77,5 / 67,3$ \\
$58,5 / 66,8^{*}$ & $63,9 / 58,5$ & $65,3 / 73,4$ \\
\hline $50,3 / 40,8^{* *}$ & $55,6 / 51,0$ & $68,0 / 66,2$ \\
\hline $31,1 / 33,3$ & $37,0 / 34,0$ & $36,0 / 40,5$ \\
$31,6 / 37,7$ & $39,3 / 34,1$ & $34,7 / 30,6$ \\
2009 & 2014 & 2019
\end{tabular}

Prozente nach Altersgruppen (65-79/80+)

\begin{tabular}{|lll}
$83,1 / 69,2^{*}$ & $87,7 / 78,9^{*}$ & $95,1 / 86,0^{* * *}$ \\
\hline $88,8 / 84,5$ & $90,9 / 83,3^{*}$ & $94,2 / 93,0$ \\
\hline $78,2 / 55,3^{* *}$ & $76,6 / 68,6$ & $79,0 / 61,0^{* * *}$ \\
$56,4 / 42,1$ & $67,3 / 54,3^{*}$ & $63,8 / 50,4^{* *}$ \\
\hline $46,6 / 42,1$ & $58,8 / 41,1^{* *}$ & $60,4 / 44,0^{* * *}$ \\
\hline $39,1 / 28,9$ & $43,7 / 18,1^{* * *}$ & $58,1 / 37,3^{* * *}$ \\
\hline $32,3 / 18,4$ & $46,7 / 21,9^{* * *}$ & $44,5 / 22,7^{* * *}$ \\
\hline $5,8 / 2,7$ & $17,6 / 9,9$ & $30,8 / 19,7^{* *}$ \\
\hline
\end{tabular}

1 =Skala (1 "lehne völlig ab" bis 5 „stimme völlig zu“); 2 = Skala ( 1 "trifft gar nicht zu“, 5 "trifft völlig zu“). Angezeigt sind nach Altersgruppen, Sprachregion und Bildungsgruppen gewichtete Daten. ." ${ }^{\prime \prime}=$ in diesem Jahr nicht erhoben. Signifikanztests zwischen Altersgruppen: T-Test bzw. Cramers V:

${ }^{* * *} p<0,001,{ }^{* *} p<0,01,{ }^{*} p<0,05$ 
In den Interviews wurden die Offliner (Personen, die das Internet nicht nutzen) auch nach ihren Gründen für die Nichtnutzung des Internets gefragt. Die in allen 3 Erhebungen konstant hoch bewerteten Gründe hierfür sind Kompliziertheit, Sicherheitsbedenken und der Aufwand. Die Kosten fallen weniger ins Gewicht. Zudem wird das Internet oft auch deswegen nicht genutzt, weil jemand anders für die ältere Person Informationen aus dem Internet abruft. Dies ist ein Grund, der über die Jahre hinweg sogar noch an Bedeutung gewonnen hat. Zwischen den Altersgruppen zeigen sich bei den beiden letzten Erhebungen keine statistisch signifikanten Unterschiede.

\section{Einflussfaktoren der Internet- nutzung}

Um herauszuarbeiten, inwieweit soziodemografische Merkmale, die subjektive Gesundheit und das Technikinteresse (Gesamtskala aus den 3 in $\bullet$ Tab. 2 vorgestellten Aussagen zur Technikeinstellung (1.1-1.3)) die Internetnutzung in den 3 Erhebungen beeinflusst haben, wurden 3 binär logistische Regressionen gerechnet (Zusatzmaterial online: Tab. 1). 2009 waren das Alter, die Bildung, das Einkommen und das Technikinteresse statistisch signifikant. Im Jahr 2014 waren es neben dem Alter, der Bildung, dem Einkommen und dem Technikinteresse auch das Geschlecht und die subjektive Gesundheit. Bei der aktuellen Erhebung 2019 waren dies ebenfalls das Alter, die Bildung, das Einkommen und das Technikinteresse. Personen, die mindestens 80 Jahre alt sind, gehören demnach seltener zu den Onlinern. Das Gleiche gilt für Personen mit einem geringeren Bildungsstatus und geringeren Einkommen. Personen mit einem höheren Technikinteresse gehören dafür eher zur Gruppe der Onliner als zur Gruppe der Offliner. Über alle 3 Erhebungen hinweg zeigen sich das Alter, die Bildung, das Einkommen und die Einstellung zur Technik als konstante Erklärungsfaktoren für die Internetnutzung. Hingegen zeigte sich 2019 kein Effekt beim Geschlecht oder der subjektiven Gesundheit.

\section{Diskussion}

Der Vergleich der 3 Bevölkerungsbefragungen von 2009, 2014 und 2019 zeigt auf, dass die digitale Transformation, die sich im Alltag in einer Zunahme der Anwendung digitaler Technologien widerspiegelt, in allen Haushalten stattfindet so auch in jenen der mindestens 65-Jährigen. Wurden 2009 oder 2014 nur wenige neue mobile Technologien wie das Smartphone genutzt, so waren es 2019 bereits mehr als die Hälfte, die ein Smartphone nutzten. Auch die Internetnutzung ist seit 2009 deutlich angestiegen und lag 2019 bei $74 \%$. Die Nutzung erklärte sich auch 2019 durch das Alter, die Bildung, das Einkommen und das Technikinteresse; Faktoren, die bereits 2009 herausgestellt wurden und auch in anderen Studien für Deutschland [9] und Europa [6] zu beobachten sind. Ferner ließen sich 2019 ähnliche Gründe für die Nichtnutzung des Internets erkennen, und auch heute werden noch nicht alle gängigen Onlineservices (z. B. Internetbanking, Kauf von Waren oder soziale Netzwerke) genutzt. Hinsichtlich des Technikinteresses und der Einstellung gegenüber dem Internet lässt auch die aktuelle Erhebung erkennen, dass zwar die Einstellungen über die Jahre eher stabil geblieben sind, aber auch jetzt noch diverse Einstellungsgruppen zu finden sind. Neben jenen mit einer positiven Einstellung gibt es auch weiterhin jene mit einer ambivalenten bis negativ geprägten Einstellung zum Internet.

Über alle 3 Befragungen hinweg zeigen sich in der multivariaten Analyse das Alter, die Bildung, das Einkommen und die Einstellung zur Technik als konstante Erklärungsfaktoren für die Internetnutzung. Somit ergeben sich auch Ende 2019 noch Unterschiede zwischen Personen im Alter von 65 bis 79 Jahren zu Personen ab 80 Jahren; auch sind individuelle Ausstattungen wie das Einkommen oder die Bildung weiterhin Ressourcen für eine Internetnutzung [6]. Hingegen zeigte sich 2019 kein Effekt beim Geschlecht oder der subjektiven Gesundheit mehr, was andeutet, dass ehemals erklärende Faktoren an Bedeutung verlieren, da heutzutage das Internet von mehr Bevölkerungsgruppen genutzt wird. Dennoch sollten gesundheitliche Aspekte bei der Internetnutzung weiterhin berücksichtigt werden, daz. B. Einschränkungen des Sehens oder Hörens direkten Einfluss auf die vollumgängliche Nutzung vom Internet nehmen können.

Auch wenn Nutzungszuwächse erkennbar sind, zeigt der Jahresvergleich auch, dass sich der digitale Graben nun v. a. innerhalb der älteren Bevölkerungsgruppe zeigt. So sind es insbesondere Personen ab 80 Jahren, die das Internet weniger nutzen und auch seltener ein Smartphone oder Tablet besitzen. Diese Nutzungsunterschiede lassen sich auch in der stationären Alterspflege erkennen, wo die Bewohnerinnen und Bewohner seltener technische Neuerungen nutzen als jüngere Personen [11, 15]. Insbesondere jene, die weniger bis gar nicht in der heutigen digitalen Welt vertreten sind, könnten sich sozial exkludiert fühlen, wenn klassische Zugänge wie ein „bedienter Postschalter" aufgrund von z.B. Sparmaßnahmen wegfallen oder wenn - wie jetzt - pandemiebedingte fehlende direkte Kontakte nicht durch digitale Lösungen kompensiert werden können [16]. Es besteht die Gefahr, dass sich diese älteren Menschen einem „Diktat des Digitalen“ [8] gegenübergestellt sehen, wenn eine Teilhabe in der heutigen Gesellschaft bedeutet, bestimmte Technologien nutzen zu müssen.

Der Vergleich der 3 Untersuchungen hinsichtlich der Verteilung von „klassischen" Technologien (z.B. Fernsehen und Radio) zu „modernen “Technologien (z.B. Smartphone und Tablet) zeigt zwar schon, dass immer mehr auch neuere Informations- und Kommunikationswege genutzt werden, aber dennoch die Nutzung des Fernsehers und des Radios relativ stabil geblieben ist. Somit kann eher vermutet werden, dass die neueren IKTGeräte nicht unmittelbar „klassische“ $\mathrm{Zu}$ gänge ersetzen, sondern ergänzen.

Werden die vorliegenden Daten mit anderen internationalen Studien verglichen, zeigen sich ähnliche Trends in der Zunahme der „modernen“ Technologien (z. B. Smartphone, Internet, Tablet), dennoch zeigt der Vergleich mit europäischen Daten [5, 6] anhand der Internetnutzung, dass die Schweiz im Vergleich z. B. zu Deutschland insgesamt höhere Nutzungszahlen bei den Personen ab 
65 Jahren aufweist. Daher sollten länderspezifische Unterschiede in der Nutzung von IKT berücksichtigt werden und die hier vorliegenden Erkenntnisse nicht 1:1 auf andere Länder übertragen werden.

Auch wenn der Zeitvergleich einen eindeutigen Trend zu "mehr Technik“ zeigt, sollte daraus nicht geschlossen werden, dass der digitale Graben in 10 bzw. 20 Jahren komplett verschwindet. Sicherlich wird sich die Nutzung der heute etablierten Alltagstechnologien ähnlich entwickeln wie die des Internets, dennoch ist davon auszugehen, dass in ca. 10 bis 20 Jahren andere Technologien den Alltag mitbestimmen werden (z. B. „virtual“ oder "augmented reality“, künstliche Intelligenz). Daher sollte innerhalb der Gerontologie nicht mehr nur gefragt werden, „wann" der digitale Graben verschwindet, sondern ,wie“ sich dieser Graben in den nächsten Jahren verschieben bzw. neu gestalten wird.

Die Gruppe der Offliner ist in ihren Merkmalen und Einstellungen heterogen, weshalb Maßnahmen immer zielgruppenbezogene oder, noch besser, individuell angepasste Lösungen berücksichtigen sollten [13]. Das Aufzeigen eines möglichen persönlichen Vorteils bei der Internetnutzung kann als anziehender Faktor dienen. Neben klassischen Schulungsangeboten sollte auch das soziale Umfeld (z. B. Familie, Freunde, Nachbarschaft) als Ressource herangezogen werden - als Impulsgeber und informelle Unterstützung [4]. Neben der reinen Vermittlung der Mediennutzungskompetenz sollte es aber auch um eine Medienbildung gehen, also die Fähigkeit, moderne Technologien nicht nur zu nutzen, sondern reflektiert zu nutzen (z. B. zu wissen, wo im Internet Gefahren lauern könnten).

\section{Limitationen}

Bei den durchgeführten Studien handelt es sich um Querschnittsuntersuchungen; Veränderungen innerhalb einer Person können daher nicht abgebildet werden. Für die weitere Forschung wäre es daher wünschenswert, einerseits individuelle Daten im Längsschnitt zu erheben, anhand derer die intraindividuelle Einstellungs- und Nutzungsveränderung be- obachtet werden könnte. Die hier vorgelegten Daten beziehen sich nur auf die Schweiz, daher sind direkte Übertragungen der Ergebnisse auf andere Länder nur für vergleichende Zwecke heranzuziehen; allfällige kulturelle Unterschiede sollten berücksichtigt werden. Zudem wurden in den 3 Befragungen Personen in Privathaushalten berücksichtigt; jedoch fehlt es an Studien zur IKT-Nutzung in den stationären Einrichtungen der Alterspflege.

\section{Fazit für die Praxis}

\section{- Auch wenn die Internetnutzung und Nutzung mobiler Endgeräte wie Smartphone und Tablet in den letzten 10 Jahren mehr und mehr in den Haushalten der älteren Menschen angekommen ist, bestehen weiterhin Nutzungsdifferenzen. \\ - Der digitale Graben verschiebt sich innerhalb der Altersgruppe der über 65-Jährigen, sodass Personen ab 80 Jahren weniger häufig z. B. das Internet nutzen als "jüngere Ältere". \\ - Die Zivilgesellschaft sollte für Schwie- rigkeiten älterer Menschen im Um- gang mit Technik sensibilisiert sein, damit Offliner nicht aus dem technik- dominierten Alltag ausgeschlossen werden.}

\section{Korrespondenzadresse

Alexander Seifert
Zentrum für Gerontologie,
Universität Zürich
Pestalozzistraße 24,
8032 Zürich, Schweiz
alexander.seifert@zfg.uzh.ch

Danksagung. Der Autor dankt Pro Senectute Schweiz für die Datennutzung und Unterstützung bei der Studie.

Funding. Open access funding provided by University of Zurich

\section{Einhaltung ethischer Richtlinien}

Interessenkonflikt. A. Seifert gibt an, dass kein Interessenkonflikt besteht.

Für diesen Beitrag wurden vom Autor keine Studien an Menschen oder Tieren durchgeführt. Für die aufge- führten Studien gelten die jeweils dort angegebenen ethischen Richtlinien.

Open Access. Dieser Artikel wird unter der Creative Commons Namensnennung 4.0 International Lizenz veröffentlicht, welche die Nutzung, Vervielfältigung, Bearbeitung, Verbreitung und Wiedergabe in jeglichem Medium und Format erlaubt, sofern Sie den/die ursprünglichen Autor(en) und die Quelle ordnungsgemäß nennen, einen Link zur Creative Commons Lizenz beifügen und angeben, ob Änderungen vorgenommen wurden.

Die in diesem Artikel enthaltenen Bilder und sonstiges Drittmaterial unterliegen ebenfalls der genannten Creative Commons Lizenz, sofern sich aus der Abbildungslegende nichts anderes ergibt. Sofern das betreffende Material nicht unter der genannten Creative Commons Lizenz steht und die betreffende Handlung nicht nach gesetzlichen Vorschriften erlaubt ist, ist für die oben aufgeführten Weiterverwendungen des $\mathrm{Ma}$ terials die Einwilligung des jeweiligen Rechteinhabers einzuholen.

Weitere Details zur Lizenz entnehmen Sie bitte der Lizenzinformation auf http://creativecommons.org/ licenses/by/4.0/deed.de.

\section{Literatur}

1. Anderson M (2019) Mobile technology and home broadband 2019. https://www.pewresearch.org/ internet/wp-content/uploads/sites/9/2019/06/ PI_2019.06.13_Mobile-Technology-and-HomeBroadband_FINAL2.pdf.Zugegriffen:02.Okt.2020

2. Francis J, Ball C, Kadylak T, Cotten SR (2019) Aging in the digital age: conceptualizing technology adoption and digital inequalities. In: Neves BB, Vetere $\mathrm{F}$ (Hrsg) Ageing and digital technology. Springer, Singapore, S35-49

3. InitiativeD21 (2020) D21-Digital-Index 2019/2020 Wie digital ist Deutschland? Initiative D21, Berlin

4. Kamin ST, Beyer A, Lang FR (2020) Social support is associated with technology use in old age. ZGerontol Geriatr 53:256-262

5. König R, Seifert A (2020) From online to offline and vice versa: change in Internet use in later life across Europe. Front Sociol 5:1-12. https://doi.org/ 10.3389/fsoc.2020.00004

6. König R, Seifert A, Doh M (2018) Internet use among older Europeans: an analysis based on SHARE data. Univers Access Inf Soc 17:621-633

7. Marston HR, Genoe R, Freeman S et al (2019) Older adults' perceptions of ICT: main findings from the technology in later life (TILL) study. Healthcare 7:86. https://doi.org/10.3390/healthcare7030086

8. Obermeier C (2020) Seniorinnen und Senioren im Kontext der digitalen Revolution: eine qualitative Untersuchung der Internetnutzung. Beltz, Weinheim

9. Quittschalle J, Stein J, Luppa M et al (2020) Internet use in old age: results of a German population-representative survey. J Med Internet Res 22:e15543. https://doi.org/10.2196/15543

10. Schelling HR, Seifert A (2010) Internet-Nutzung im Alter. Gründe der (Nicht-)Nutzung von Informations- und Kommunikationstechnologien (IKT) durch Menschen ab 65 Jahren in der Schweiz. Zentrum für Gerontologie, Zürich

11. Schlomann A, Seifert A, Zank S, Rietz C (2020) Assistive technology and mobile ICT usage among oldest-old cohorts: comparison of the oldest-old in 
private homes and in long-term care facilities. Res Aging 42:163-173

12. Schmidt L, Wahl H-W (2019) Alter und Technik. In: Hank K, Schulz-Nieswandt F, Wagner M, Zank S (Hrsg) Alternsforschung. Nomos, Baden-Baden, $S$ 537-556

13. Seifert A (2016) Internetkompetenzen im Alter Überlegungen zur Förderung von Medienkompetenzen im Alter. Merz 2016:66-71

14. Seifert A,AckermannT, Schelling HR(2020)Digitale Senioren III - 2020/Nutzung von Informationsund Kommunikationstechnologien (IKT) durch Menschen ab 65 Jahren in der Schweiz. Zentrum für Gerontologie, Zürich

15. Seifert A, Cotten SR (2020) In care and digitally savvy? Modern ICT use in long-term care institutions. Educ Gerontol 46:473-485

16. Seifert A, Cotten SR, Xie B (2020) A double burden of exclusion? Digital and social exclusion of older adults in times of COVID-19.J Gerontol B Psychol Sci Soc Sci.https://doi.org/10.1093/geronb/gbaa098

17. Seifert A, Schelling HR (2015) Digitale Senioren. Nutzung von Informations- und Kommunikationstechnologien (IKT) durch Menschen ab 65 Jahren in der Schweiz im Jahr 2015. ProSenectute, Zürich

18. Seifert A, Schelling HR (2016) Alt und offline?: Befunde zur Nutzung des Internets durch Menschen ab 65 Jahren in der Schweiz. Z Gerontol Geriatr 49:619-625

Die Anaesthesiologie Die Innere Medizin Die Pathologie

Die Chirurgie Die Kardiologie Pneumologie

Die Dermatologie Die MKG-Chirurgie Die Psychotherapie

Die Diabetologie Die Nephrologie Die Radiologie

Die Gastroenterologie Opie Ophthalmologie Die Unfallchirurgie

Die Gynäkologie Die Orthopädie Die Urologie

\section{Und wenn der Facharzt eine Frau ist?}

Im Zuge einer breit angelegten Gleichstellungsinitiative des Springer Medizin Verlages werden die bisherigen, auf männlichen Berufsbezeichnungen basierenden Titel durch neue, auf die Fachgebiete bezogene Titel ersetzt. Damit die Fachzeitschriften auch außen wieder up to date sind.

Die Fachzeitschriften des Springer Medizin Verlags gehen mit den berufs- und gesellschaftspolitischen Entwicklungen und erscheinen ab ihren Juni- bzw. Juliausgaben mit neuen Titeln. Das Titelkonzept trägt auch einem geänderten Umgang mit Sprache Rechnung und verfolgt das Prinzip, dass zukünftig das Fachgebiet und nicht mehr die männliche Berufsbezeichnung im Fokus steht. So wird aus "Der Internist" ab Juni die Fachzeitschrift „Die Innere Medizin", oder „Der Chirurg" heißt künftig „Die Chirurgie”. Diese Änderungen betreffen 21 Zeitschriften, die bei Springer Medizin erscheinen und Teil der Fachverlagsgruppe Springer Nature sind. Sie sind eine von vielen Maßnahmen innerhalb Springer Nature. Auf dem Portal Springer Medizin: www.springermedizin.de/titelupdate findet sich eine Übersicht aller betreffenden Zeitschriften samt detaillierten Erklärungen zu Motiven und Hintergründen.

Die Medizin ist schon lange nicht mehr männlich, wie es noch zu Gründungszeiten einiger Springer Fachzeitschriften der Fall war. Seit 1999 machen Frauen eine klare Mehrheit unter den Studierenden aus, im Jahr 2020 belief sich ihr Anteil auf knapp zwei Drittel. Im Berufsalltag liegt der Frauenanteil bei fast $50 \%$, in einigen Bereichen auch deutlich höher. "Diesem Wandel in der Medizin werden wir Rechnung tragen", erklärt Dr. Paul Herrmann, Director Journals und ePublishing von Springer Medizin. „Als einer der führenden Verlage in der Wissenschaftskommunikation geben wir jetzt ein klares Signal und machen Geschlech- tergerechtigkeit in der Medizin an dieser Stelle sichtbar."

Unverändert bleibt bei den Springer Fachzeitschriften die inhaltliche Ausrichtung; diese unterliegen weiterhin einer praxisrelevanten Aufbereitung und Kommunikation von Forschungsergebnissen in den Fachgebieten. Ebenso garantiert wird eine kontinuierliche Sichtbarkeit innerhalb der internationalen Wissenschaftscommunity: Ein intensiver Austausch mit relevanten internationalen Wissenschaftsdatenbanken sowie deren Offenheit und Support für das Thema garantiert, dass der Wechsel der Titel ohne nachteilige Konsequenzen verlaufen wird und eine Listung oder ein vorhandener Impact Factor bestehen bleibt.

Mehr zur Titeländerung finden Sie auf www.springermedizin.de/titelupdate oder direkt über diesen QR-Code:

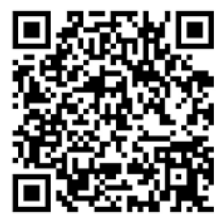

